\section{Glukokortikoide und Kataraktrisiko}

\begin{abstract}
Inhalative Glukokortikoide sind gut verträgliche Medikamente. In höheren Dosen können sie jedoch die selben Nebenwirkungen wie bei systemischer Gabe hervorrufen. In einer Fall-Kontrollstudie sollte dies für die Katarakt quantifiziert werden - eine Nebenwirkung, die für systemische Glukokortikoide dokumentiert ist.
\end{abstract}

E pidemiologen aus London recherchierten in der General Practice Research Database (GPRD), einer der größten Datenbanken für Erkrankungen und Medikamenteneinnahme in Großbritannien, die Krankenberichte von 15.479 Patienten mit Katarakt und verglichen sie mit den Daten von 15.479 Kontrollpersonen ohne Katarakt. Das mittlere Alter der Patienten betrug 75 Jahre. In der Kataraktgruppe hatten 11,4\% der Patienten im Laufe ihres Lebens wegen einer Lungenerkrankung (Asthma, COPD) ein inhalatives Glukokortikoid benutzt, in der Kontrollgruppe waren es nur 7,5\%. Zwischen der Applikation eines inhalativen Glukokortikoids und einer Katarakt ergab sich damit ohne Einbeziehung weiterer Faktoren eine Odds ratio (OR) von 1,58. Nach Adjustierung hin-

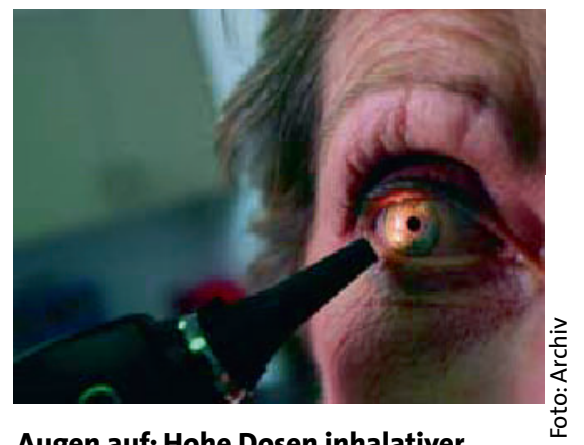

Augen auf: Hohe Dosen inhalativer Glukortikoide sind über einen längeren Zeitraum gegeben mit einem erhöhten Kataraktrisiko verbunden.

sichtlich der Einnahme systemischer Glukokortikoide und der Häufigkeit der Arztbesuche fiel die OR auf 1,10.

Diese adjustierte OR zeigte allerdings einen deutlichen dosisabhängigen Effekt: Für tägliche Inhalations- mengen bis zu $400 \mu \mathrm{g}$ ergab sich eine OR von 0,99 , für tägliche Inhalationsmengen zwischen 400 und $800 \mu \mathrm{g}$ sowie für Mengen zwischen 800 und $1600 \mu \mathrm{g}$ eine OR von 1,18 und für sehr hohe Tagesdosen mit mehr als $1.600 \mu \mathrm{g}$ Glukokortikoid eine OR von 1,69. Dieser Zusammenhang war umso ausgeprägter, je länger inhaliert wurde. Das am häufigste inhalierte Glukokortikoid war Beclometason. Budesonid und Fluticason als neuere Substanzen fielen dagegen bei dieser schon älteren Patientenkohorte kaum ins Gewicht.

Fazit: Die Applikation hoher Dosen inhalativer Glukokortikoide über einen längeren Zeitraum korrelierte in dieser retrospektiven Erhebung positiv mit einem erhöhten Kataraktrisiko. Augenärztliche Kontrollen können daher auch unter inhalativen Glukokortikoiden indiziert sein. Die Studie belegt aber zugleich, dass das Risiko vor allem auf der (intermittierenden) systemischen Glukokortikoidgabe beruht.

$b k$

Smeeth L et al. A population based casecontrol study of cataract and inhaled corticosteroids. Br J Ophthalmol 2003; 87: 1247-51

\title{
Was tun, wenn Asthma schlimmer wird?
}

\section{Stabil eingestellte Asthmapatienten sollten bei einer Verschlech- terung der Symptomatik die Dosis ihres inhalativen Glukokortikoids erhöhen. Diese allgemein anerkannte Empfehlung wird durch die Ergebnisse einer englischen Studie in Frage gestellt.}

stellten Dosieraerosolen, die entweder tatsächlich die doppelte Glukokortikoiddosis oder die bisherige Dosis enthielten. Wenn sich die Symptomatik weiter bis zu einem vorher definierten Punkt verschlechterte, nahmen die Probanden täglich $30 \mathrm{mg}$ Prednisolon oral ein.

Innerhalb eines Jahres brachten 207 (53\%) der Patienten den verblindeten Studieninhalator zum Einsatz. Zwischen der Verum- und der Plazebogruppe ergaben sich keine Unterschiede hinsichtlich Peak-Flow-Werten und Sym- ptomscore. Zum letzten Mittel der oralen Glukokortikoideinnahme griffen schließlich 22 von 192 Patienten (11\%) aus der Verumgruppe und 24 von 198 (12\%) aus der Plazebogruppe. Dieser Unterschied zwischen den Gruppen war nicht signifikant.

Fazit: Die auch in offiziellen Leitlinien enthaltene Empfehlung, bei Auftreten bzw. Verschlimmerung der Asthmasymptome die Dosis inhalativer Glukokortikoide zu erhöhen, wird durch die hier vorgestellte kontrollierte Studie nicht gestützt. Eine Bestätigung der Resultate durch eine Untersuchung mit einer größeren Teilnehmerzahl ist wünschenswert.

Harrison TW et al. Doubling the dose of inhaled corticosteroid to prevent asthma exacerbations: randomised controlled trial. Lancet 2004; 363: 271-5 